\title{
Asociación de riesgo entre periodontitis y preeclampsia. una revisión sistemática
}

\section{retrospectiva}

\author{
Risk association between periodontitis and preeclampsia. a retrospective systematic review \\ Associação de risco entre periodontite e pré-eclâmpsia. uma revisão sistemática retrospectiva
}

Recibido: 08/08/2021 | Revisado: 10/08/2021 | Acepto: 11/08/2021 | Publicado: 15/08/2021

\author{
Andrés Santiago Cáceres Alvear \\ ORCID: https://orcid.org/0000-0002-2540-0882 \\ Universidad de Cuenca, Ecuador \\ E-mail: santiago.caceres@ucuenca.edu.ec \\ Damián Alejandro Garcés García \\ ORCID: https://orcid.org/0000-0001-6715-8046 \\ Universidad de Cuenca, Ecuador \\ E-mail: alejandro.garces@ucuenca.edu.ec \\ Cristina Paola Carpio Cedillo \\ ORCID: https://orcid.org/0000-0002-4115-4175 \\ Universidad de Cuenca, Ecuador \\ E-mail: cristina.carpioc@ucuenca.edu.ec
}

\begin{abstract}
Resumen
Objetivo. La presente revisión sistemática tiene como finalidad evaluar la literatura disponible sobre la asociación de riesgo entre periodontitis y preeclampsia. Metodología. Se realizó una búsqueda de artículos científicos que estudiaron la asociación entre periodontitis y preeclampsia, se utilizaron como motores de búsqueda las plataformas MEDLINE (PubMed) y Scopus, se seleccionaron estudios de tipo caso-control que reportaron resultados como el OR (Odds ratio), definiciones claras y criterios de diagnóstico adecuados para periodontitis y preeclampsia. La revisión se realizó de acuerdo con las directrices PRISMA (Preferred Reporting Items for Systematic Reviews and Meta analysis). Además, para controlar la calidad de los estudios incluidos se utilizó la declaración STROBE (Strengthening the Reporting of Observational Studies in Epidemiology). Resultados. Aplicados los criterios de inclusión y exclusión se obtuvieron 15 estudios caso-control que fueron incluidos en la revisión. De los 15 estudios incluidos, $13(86,67 \%)$ transmisión una asociación positiva entre la periodontitis y el desarrollo de preeclampsia. La edad, la paridad y el tabaquismo fueron las variables más controladas en cada estudio. Conclusión. La periodontitis fue un factor de riesgo para preeclampsia, enfatizando la importancia de la atención periodontal en los programas prenatales. Registro de revisión sistemática: PROSPERO CRD42020205855.
\end{abstract}

Palabras clave: Enfermedad periodontal; Preeclampsia; Periodontitis; Efectos adversos del embarazo; Factores de riesgo.

\begin{abstract}
Objective. The present systematic review aims to evaluate the available literature on the risk association between periodontitis and preeclampsia. Methodology. A search was carried out for scientific articles that studied the association between periodontitis and preeclampsia, the MEDLINE (PubMed) and Scopus platforms were used as search engines, case-control studies were selected that reported results such as the OR (Odds ratio), clear definitions and appropriate diagnostic criteria for periodontitis and preeclampsia. The review was performed according to the PRISMA (Preferred Reporting Items for Systematic Reviews and Meta analysis) guidelines. In addition, the STROBE (Strengthening the Reporting of Observational Studies in Epidemiology) statement was used to control the quality of the included studies. Results. Applying the inclusion and exclusion criteria, 15 case-control studies were obtained and included in the review. Of the 15 included studies, 13 (86.67\%) showed a positive association between periodontitis and the development of preeclampsia. Age, parity, and smoking were the most controlled variables in each study. Conclusion. Periodontitis was a risk factor for preeclampsia, emphasizing the importance of periodontal care in prenatal programs. Systematic review registration: PROSPERO CRD42020205855.
\end{abstract}

Keywords: Periodontal disease; Preeclampsia; Periodontitis; Adverse effects of pregnancy; Risk factors.

\section{Resumo}

Alvo. A presente revisão sistemática tem como objetivo avaliar a literatura disponível sobre a associação de risco entre periodontite e pré-eclâmpsia. Metodologia. Foi realizada uma busca de artigos científicos que estudaram a associação entre periodontite e pré-eclâmpsia, as plataformas MEDLINE (PubMed) e Scopus foram utilizadas como motores de busca, foram selecionados estudos de caso-controle que relataram resultados como o OR (Odds ratio), 
claro definições e critérios diagnósticos apropriados para periodontite e pré-eclâmpsia. A revisão foi realizada de acordo com as diretrizes PRISMA (Preferred Reporting Items for Systematic Reviews and Meta analysis). Além disso, a declaração STROBE () foi usada para controlar a qualidade dos estudos incluídos. Resultados. Aplicando os critérios de inclusão e exclusão, foram obtidos 15 estudos caso-controle que foram incluídos na revisão. Dos 15 estudos incluídos, $13(86,67 \%)$ transmitiram associação positiva entre periodontite e o desenvolvimento de préeclâmpsia. Idade, paridade e tabagismo foram as variáveis mais controladas em cada estudo. Conclusão. A periodontite foi um fator de risco para pré-eclâmpsia, enfatizando a importância dos cuidados periodontais nos programas de pré-natal. Registro de revisão sistemática: PROSPERO CRD42020205855.

Palavras-chave: Doença periodontal; Pré-eclâmpsia; Periodontite; Efeitos adversos da gravidez; Fatores de risco.

\section{Introducción}

La enfermedad periodontal es una afección inflamatoria crónica de origen multifactorial caracterizada por un biofilm disbiótico, que provoca la destrucción progresiva de los tejidos de sostén del diente (Casas, 2020). En 2017 en el World Workshop on the Classification of Periodontal and Peri-implant Diseases and Conditions se definió un caso de periodontitis como: pérdida de inserción clínica (CAL) interdentaria en dos o más dientes no adyacentes, o bien CAL vestibular $\geq 3$ mm con bolsas de >3 mm en dos o más dientes (Herrera et al., 2018).

La incidencia y la prevalencia de la enfermedad periodontal han aumentado en los últimos 30 años, siendo ahora la doceava enfermedad más prevalente en el mundo; además, las mujeres tienen un 13\% más de riesgo de padecer de enfermedad, los datos epidemiológicos han expuesto que la enfermedad periodontal es una epidemia creciente (Nocini et al., 2020). La periodontitis aumenta con la edad, desde la adolescencia hasta el adulto mayor y es más común en países de altos ingresos (Nazir et al., 2020). En el Reino Unido y en Estados Unidos afecta a cerca de la mitad de los adultos y al $60 \%$ de la población mayor a 65 años, disminuyendo la calidad de vida de estas personas, por lo que es considerado un problema de salud pública (Casas, 2020), (Carvajal, 2016).

Según la Organización Mundial de la Salud (2014): "Los trastornos hipertensivos del embarazo son una causa importante de morbilidad y mortalidad maternas y perinatales, afectando a alrededor del 10\% de mujeres embarazadas en todo el mundo". En Latinoamérica, la morbilidad perinatal representa del 8 al 45\% y la mortalidad perinatal del 1 al 33\% (CondoBaque et al., 2018). La preeclampsia es una forma de hipertensión arterial en el embarazo que perjudica tanto a la madre como al recién nacido (Varshney \& Gautam, 2014). Constituye junto a la eclampsia la tercera causa de muerte materna con al menos el 30\% de los casos (Condo-Baque et al., 2018). En la revisión sistemática y metaanálisis de Macedo et al., (2020) que analizó la prevalencia de preeclampsia y eclampsia en 70 estudios en los últimos 50 años, reveló una prevalencia del 6,7\% (IC del 95\%: 5,8-7,6), además se encontró una mayor prevalencia en países de ingresos bajos y medios $(11,5 \%$, IC del 95\%: 7,8-15,8 y 10,6\%, IC del 95\%: 6,05-16,2 respectivamente).

La preeclampsia se presenta con la típica tríada de: hipertensión, proteinuria, hinchazón o edema primordialmente en manos y cara, cefalea, etc (Varshney \& Gautam, 2014). Un caso de preeclampsia se presenta como una paciente con una presión arterial (PA) mayor a 140/90 mmHg después de las 20 semanas de gestación en dos ocasiones distanciadas por un intervalo de tiempo de 4 horas y proteinuria $>300 \mathrm{mg}$ en una muestra de orina de 24 horas o un índice de proteína creatinina $>0,3 \mathrm{mg}$ o un resultado $\geq 2+$ en tiras reactivas (Pereira Calvo et al., 2020).

Numerosas investigaciones han acentuado el concepto de que la periodontitis es considerada un factor de riesgo para resultados adversos en el embarazo como por ejemplo el parto prematuro, el bajo peso al nacer y la preeclampsia (Desai et al., 2015). Bogges et al., (2003) en su estudio de cohorte publicado en el año 2003, que incluyó 1115 mujeres embarazadas demostró por primera vez la relación entre enfermedad periodontal y riesgo de desarrollar preeclampsia. Sin embargo, todavía existe mucha controversia al respecto (Lohsoonthorn et al., 2009).

Uno de los mecanismos principales en la patogenia de la preeclampsia es el daño del endotelio vascular (Boggess et 
al., 2003). Sin embargo, el factor iniciador de este proceso aún es desconocido (Huang et al., 2014). Se ha planteado que los patógenos periodontales pueden llegar a la región úteroplacentaria y producir inflamación o estrés oxidativo y la subsiguiente disfunción endotelial (aterosis aguda), lo que llevaría a la preeclampsia (Huang et al., 2014). Esto podría ser apoyado por la investigación de Barak et al., (2007) que identificó por primera vez la presencia de patógenos periodontales (Actinobacillus actinomycetemcomitans, Fusobacterium nucleatum, Porphyromonas gingivalis, Prevotella intermedia, Tannerella forsythia y Treponema denticola) en la placenta humana. Dichos patógenos fueron más prevalentes en el grupo de mujeres preeclámpticas en comparación con el grupo de controles normotensos (Barak et al., 2007). De modo que, la asociación entre periodontitis y la preeclampsia puede adjudicarse a la diseminación frecuente de patógenos periodontales a los tejidos deciduales mediante una bacteriemia transitoria (Desai et al., 2015).

Por lo tanto, el presente estudio tiene como finalidad evaluar la literatura disponible sobre la posible asociación de riesgo entre la periodontitis y la preeclampsia.

\section{Metodología}

\section{Protocolo y registro}

La presente revisión sistemática se realizó de acuerdo con las directrices PRISMA (Preferred Reporting Items for Systematic Reviews and Meta analysis) (Urrutia \& Bonfill, 2010). El protocolo fue registrado en el sitio web PROSPEROtm el día 11 de febrero de 2021, pudiendo acceder a él mediante el siguiente enlace:

https://www.crd.york.ac.uk/PROSPERO/display_record.php?RecordID=230723" th

\section{Criterios de elegibilidad}

Criterios de inclusión: se incluyeron estudios de casos y controles que cumplieron con los siguientes requisitos: redactados en inglés y español, con mujeres en edades comprendidas entre los 15 y 46 años. Estudios que analizan la asociación entre periodontitis y preeclampsia con una definición clara y criterios de diagnóstico correspondientes para estas patologías y que reporten un OR (Odds ratio).

Criterios de exclusión: se excluyeron estudios que incluyeron pacientes con enfermedades sistémicas, que no reportaron OR y estudios complementarios, duplicados o con resultados ambiguos.

\section{Estrategia de búsqueda}

Se realizó una búsqueda a través de las plataformas MEDLINE (PubMed) y Scopus, sin restricciones de fechas, seleccionando los resultados desde sus registros más antiguos hasta el día 20 de septiembre de 2020. Además, solo se incluyeron resultados de estudios redactados en los siguientes idiomas: inglés y español.

Para la búsqueda se obtuvieron palabras clave en MESH y se realizaron combinaciones utilizando operadores booleanos, incluidos: Y (AND) y paréntesis, con los siguientes términos: periodontal disease, periodontitis, periodontal condition, periodontal health, periodontal pocket, attachment loss, preeclampsia, hypertension pregnancy, case-control. Esta estrategia de búsqueda se adaptó para ser utilizada en ambas bases de datos electrónicas.

\section{Proceso de selección de los estudios y recopilación de datos}

Dos autores de la revisión (SC \& DG) seleccionaron de forma independiente los títulos y / o resúmenes recuperados de las fuentes de las bases de datos electrónicas para identificar los estudios potencialmente elegibles. En una hoja de cálculo (Microsoft Office Exceltm) se documentaron los siguientes datos de cada estudio seleccionado: iniciales del investigador responsable, motor de búsqueda, autor del artículo, año de publicación, título completo del artículo, tamaño de la muestra, OR, 
conclusión principal, enlace del artículo. Los mismos autores recuperaron el texto completo de los estudios seleccionados y obtuvieron las definiciones de periodontitis y preeclampsia para cada estudio. Los estudios que cumplieron con los criterios de selección establecidos se evaluaron de forma independiente para determinar su elegibilidad, y los desacuerdos sobre la inclusión o exclusión de cualquier estudio se resolvieron con la participación de un tercer autor (CC).

Para controlar la calidad de los estudios incluidos se utilizó la declaración STROBE (Strengthening the Reporting of Observational studies in Epidemiology) (Vandenbroucke et al., 2009), los estudios que cumplieron con un 50\% o más de estas directrices fueron incluidos en esta revisión sistemática.

\section{Resultados}

\section{Selección de estudios}

Como resultado de la estrategia de búsqueda se recopilaron 539 resultados en PubMed y 204 en Scopus que analizarán la posible asociación entre periodontitis y preeclampsia. Se realizó un primer screening en donde se descartaron 476 y 152 respectivamente por ser resultados duplicados. Posteriormente se obtuvieron 63 artículos de PubMed y 52 de Scopus, y de estos se excluyeron 38 y 29 respectivamente por presentar títulos y/o resúmenes irrelevantes para la revisión, a continuación se eliminaron los estudios repetidos en ambas bases de datos, obteniendo un total de 30 estudios, mediante el análisis completo de estas publicaciones se eliminaron 15 artículos por no cumplir con los criterios de selección, dando como resultado una base de datos de 15 artículos, los cuales al ser sometidos a las directrices STROBE (Vandenbroucke et al., 2009), dieron un porcentaje mayor al 50\%, siendo incluidos todos los artículos para la revisión (Figura 1).

Figura 1. Diagrama de flujo del proceso de selección de los estudios.

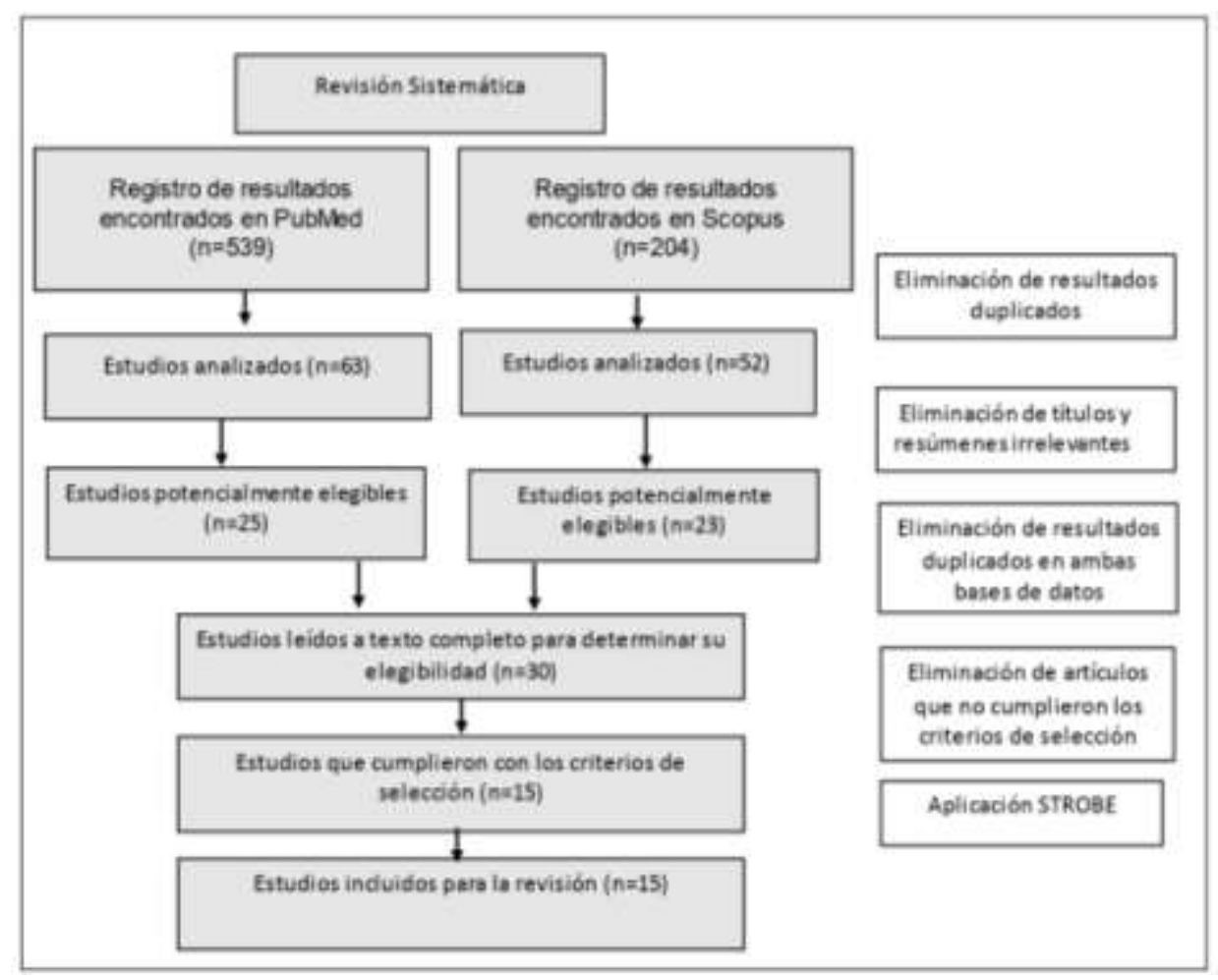

Fuente: Autores.

\section{Síntesis de datos}

En la Tabla 1 podemos observar que los estudios analizados en la presente revisión fueron artículos publicados entre los años 2004 - 2015. Comprendiendo una muestra total de 5991 sujetos, la edad media de las poblaciones estudiadas entre 
casos y controles ronda los 27,59 años de edad ( \pm 5,27). La mayoría de los estudios se realizaron en el continente asiático (46,67\%), seguido de estudios realizados en Sudamérica (33,33\%), finalmente existieron estudios del continente europeo y norteamericanos $(6,67 \%)$ respectivamente. Además, en la Tabla 1 observamos cuáles fueron los criterios diagnósticos para determinar la periodontitis y preeclampsia. De estos datos se puede evidenciar que la definición más usada de periodontitis fue $\mathrm{PD} \geq 4 \mathrm{~mm}$ y $\mathrm{CAL} \geq 3 \mathrm{~mm}$ en el mismo sitio en al menos 4 dientes, mientras que preeclampsia se definió como la presión arterial $\geq 140 / 90 \mathrm{mmHg}$ con proteinuria asociada.

Tabla 1. Características de los estudios incluídos.

\begin{tabular}{|c|c|c|c|c|c|c|c|}
\hline \multirow[t]{2}{*}{ ID } & \multirow[t]{2}{*}{ Autor } & \multirow[t]{2}{*}{ País } & \multirow[t]{2}{*}{$\begin{array}{c}\text { Total } \\
\text { muestra }\end{array}$} & \multicolumn{2}{|c|}{ Edad media (DE) } & \multirow[t]{2}{*}{ Definición de periodontitis } & \multirow[t]{2}{*}{ Definición de preeclampsia } \\
\hline & & & & Casos & Controles & & \\
\hline 1 & $\begin{array}{l}\text { (Pralhad } \\
\text { et al., 2013) }\end{array}$ & India & 200 & $\begin{array}{l}27,1( \pm \\
4,5)\end{array}$ & $26,1( \pm 3,8)$ & $\begin{array}{l}\text { Cualquiera de los siguientes } \\
\text { casos: } 1 \text { ) OHI> } 3 \text { (mala); 2) } \\
\text { GI> } 1 \text { (inflamación gingival } \\
\text { de moderada a grave); } 3 \text { ) } \\
\text { PD media >4 mm; y 4) } \\
\text { CAL > } 3 \mathrm{~mm} .\end{array}$ & $\begin{array}{l}\text { Hipertensión sostenida } \\
\text { después de las } 20 \text { semanas de } \\
\text { gestación con o sin } \\
\text { proteinuria asociada. PA } \\
\geq 140 / 90 \mathrm{mmHg} \text {. }\end{array}$ \\
\hline 2 & $\begin{array}{l}\text { (Desai } \\
\text { et al., 2015) }\end{array}$ & India & 1240 & \multicolumn{2}{|c|}{ No reporta } & $\begin{array}{l}\mathrm{PD} \geq 4 \mathrm{~mm} \text { y } \mathrm{CAL} \geq 3 \mathrm{~mm} \\
\text { en el mismo sitio en al } \\
\text { menos cuatro dientes }\end{array}$ & $\begin{array}{l}\mathrm{PA} \geq 140 / 90 \mathrm{mmHg} \text { en dos } \\
\text { ocasiones separadas después } \\
\text { de la semana } 20 \mathrm{de} \text { gestación } \\
\text { y proteinuria } \geq 0,30 \mathrm{~g} / \mathrm{dl} \text { en } \\
\text { recogida de orina de } 24 \text { horas. }\end{array}$ \\
\hline 3 & $\begin{array}{l}\text { (Moura da } \\
\text { Silva et al., } \\
\text { 2012) }\end{array}$ & Brasil & 574 & \multicolumn{2}{|l|}{$24,4 \S^{*}$} & $\begin{array}{l}\geq 4 \text { dientes con } \geq 1 \text { sitios con } \\
\text { un } \mathrm{PD} \geq 4 \mathrm{~mm} \text { y } \mathrm{CAL} \geq 3 \\
\mathrm{~mm} \text { en el mismo sitio. }\end{array}$ & $\begin{array}{l}\text { PA sistólica } \geq 140 \mathrm{mmHg} \text { o } \\
\text { PA diastólica } \geq 90 \mathrm{mmHg} \text { y } \\
\text { proteinuria } \geq 300 \mathrm{mg} / 24 \mathrm{~h} \text { o } \\
\text { 2+ en tiras reactivas, } \\
\text { desarrolladas después de la } \\
\text { semana } 20 \text { de gestación en } \\
\text { mujeres previamente } \\
\text { normotensas. }\end{array}$ \\
\hline 4 & $\begin{array}{l}\text { (Taghzouti } \\
\text { et al., 2012) }\end{array}$ & Canadá & 337 & \multicolumn{2}{|c|}{ No reporta } & $\begin{array}{l}\geq 4 \text { sitios con } \mathrm{PD} \geq 5 \mathrm{~mm} \text { y } \\
\text { una } C A L \geq 3 \mathrm{~mm} \text { en los } \\
\text { mismos sitios. }\end{array}$ & $\begin{array}{l}\mathrm{PA} \geq 140 / 90 \mathrm{mmHg} \text { y } \geq 1+ \\
\text { proteinuria después de las } 20 \\
\text { semanas de gestación. }\end{array}$ \\
\hline 5 & $\begin{array}{l}\text { (Ha et al., } \\
2011)\end{array}$ & $\begin{array}{l}\text { Corea del } \\
\text { Sur }\end{array}$ & 64 & $\begin{array}{l}32,69 \\
( \pm 5,30 \\
)\end{array}$ & $32,69( \pm 4,40)$ & $\begin{array}{l}\text { CAL } \geq 3,5 \mathrm{~mm} \geq 4 \text { sitios } \\
\text { que no están en el mismo } \\
\text { diente. }\end{array}$ & $\begin{array}{l}\mathrm{PA}>140 / 90 \mathrm{mmHg} \text { en dos } \\
\text { ocasiones distintas y } \\
\text { proteinuria } \geq 1+\text { en una } \\
\text { muestra aleatoria de orina. }\end{array}$ \\
\hline 6 & $\begin{array}{l}\text { (Politano } \\
\text { et al., 2011) }\end{array}$ & Brasil & 116 & $\begin{array}{l}28,62 \\
( \pm 6,93\end{array}$ & $24,69( \pm 5,37)$ & $\begin{array}{l}\geq 2 \text { sitios con } \mathrm{PD} \geq 4 \mathrm{~mm}, \\
\mathrm{CAL}(\geq 4 \mathrm{~mm}) \text { y BOP. }\end{array}$ & $\begin{array}{l}\text { PA sistólica } \geq 140 \mathrm{mmHg} \text { y } / \text { o } \\
\text { PA diastólica } \geq 90 \mathrm{mmHg} \text { y } \\
\text { proteinuria } \geq 300 \mathrm{mg} / 24 \mathrm{~h} .\end{array}$ \\
\hline 7 & $\begin{array}{l}\text { (Sayar } \\
\text { et al., 2011) }\end{array}$ & Irán & 210 & No repo & & $\begin{array}{l}\mathrm{CAL} \geq 3 \mathrm{~mm} \text { en al menos el } \\
30 \% \text { de las áreas. }\end{array}$ & $\begin{array}{l}\mathrm{PA} \geq 140 / 90 \mathrm{mmHg} \text { y } \\
\text { proteinuria }+1 .\end{array}$ \\
\hline 8 & $\begin{array}{l}\text { (Shetty } \\
\text { et al., 2010) }\end{array}$ & India & 130 & $26,8( \pm 3$ & 7) $\S$ & $\begin{array}{l}\text { CAL de } \geq 3 \mathrm{~mm} \text { y una PD } \\
\text { de } \geq 4 \mathrm{~mm} .\end{array}$ & $\begin{array}{l}\text { PA }>140 / 90 \text { mmHg después } \\
\text { de la semana } 20 \text { de gestación } \\
\text { como una proteinuria }>300 \\
\text { mg en una muestra de orina } \\
\text { de } 24 \text { horas. }\end{array}$ \\
\hline 9 & $\begin{array}{l}\text { (Lohsoonth } \\
\text { orn et al., } \\
2009 \text { ) }\end{array}$ & Tailandia & 300 & No repo & & $\begin{array}{l}\text { Periodontitis grave }(\geq 2 \\
\text { dientes no adyacentes con } \\
\text { sitios interproximales que } \\
\text { muestran CAL } \geq 6 \mathrm{~mm} \text { y PD } \\
\geq 4 \mathrm{~mm}) \text {; periodontitis } \\
\text { moderada ( } \geq 2 \text { dientes no } \\
\text { adyacentes con sitios } \\
\text { interproximales que } \\
\text { muestran CAL } \geq 5 \mathrm{~mm} \text { y PD } \\
\geq 4 \mathrm{~mm}) ; \text { periodontitis leve } \\
(\geq 1 \text { dientes con sitios }\end{array}$ & $\begin{array}{l}\text { PA sistólica } \geq 140 \text { o la PA } \\
\text { diastólica } \geq 90 \mathrm{mmHg} \text { (con } \\
\text { lecturas con una separación } \\
\text { de } \geq 6 \mathrm{~h} \text { ) y proteinuria } \geq 30 \mathrm{mg} \\
\text { / dl o } 1+\text { en una tira reactiva } \\
\text { de orina (en } \geq 2 \text { muestras } \\
\text { aleatorias recolectadas con } \geq 4 \\
\text { h de diferencia). }\end{array}$ \\
\hline
\end{tabular}




\begin{tabular}{|c|c|c|c|c|c|c|c|}
\hline & & & & & & $\begin{array}{l}\text { interproximales que } \\
\text { muestran } C A L \geq 4 \mathrm{~mm} \text { y } \mathrm{PD} \\
\geq 4 \mathrm{~mm} \text { ). }\end{array}$ & \\
\hline 10 & $\begin{array}{l}\text { (Siqueira } \\
\text { et al., 2008) }\end{array}$ & Brasil & 1206 & \multicolumn{2}{|c|}{$25,87( \pm 5,97) \S$} & $\begin{array}{l}\mathrm{PD} \geq 4 \mathrm{~mm} \text { y } \mathrm{CAL} \geq 3 \mathrm{~mm} \\
\text { en el mismo sitio en al } \\
\text { menos cuatro dientes. }\end{array}$ & $\begin{array}{l}\mathrm{PA}>140 / 90 \mathrm{mmHg} \text { en dos } \\
\text { ocasiones separadas después } \\
\text { de la semana } 20 \text { de gestación } \\
\text { y proteinuria } \geq 1 \text { o } \geq 0,30 \mathrm{~g} / \mathrm{dl} \text {, } \\
\text { en dos muestras de orina } \\
\text { separadas tomadas con } 6 \\
\text { horas de diferencia. }\end{array}$ \\
\hline 11 & $\begin{array}{l}\text { (Kunnen } \\
\text { et al., 2007) }\end{array}$ & $\begin{array}{l}\text { Países } \\
\text { Bajos }\end{array}$ & 52 & $\begin{array}{l}29,5 \\
( \pm 5,1)\end{array}$ & $31,7( \pm 4,2)$ & $\begin{array}{l}\geq 15 \text { sitios dentales con una } \\
\text { PD de } 4 \text { mm y BOP. }\end{array}$ & $\begin{array}{l}\text { PA diastólica } \geq 90 \mathrm{mmHg} \\
\text { medida en dos ocasiones con } \\
\text { al menos } 4 \mathrm{~h} \text { de diferencia en } \\
\text { combinación con proteinuria } \\
\text { ( } \geq 300 \mathrm{mg} / 24 \mathrm{~h} \text { o } 2+\text { tira } \\
\text { reactiva) que se desarrolla } \\
\text { después de una edad } \\
\text { gestacional de } 20 \text { semanas en } \\
\text { una mujer previamente } \\
\text { normotensa. }\end{array}$ \\
\hline 12 & $\begin{array}{l}\text { (Cota et al., } \\
\text { 2006) }\end{array}$ & Brasil & 1067 & 26,5 & 5) $\S$ & $\begin{array}{l}\geq 4 \text { dientes con uno o más } \\
\text { sitios con } \mathrm{PD} \geq 4 \mathrm{~mm} \text { y } \\
\mathrm{CAL} \geq 3 \mathrm{~mm} \text { en el mismo } \\
\text { sitio. }\end{array}$ & $\begin{array}{l}\mathrm{PA}>140 / 90 \mathrm{mmHg} \text { y } \\
\text { proteinuria } \geq 1+\text { después de } \\
\text { las } 20 \text { semanas de gestación. }\end{array}$ \\
\hline 13 & $\begin{array}{l}\text { (Contreras } \\
\text { et al., 2006) }\end{array}$ & Colombia & 373 & $\begin{array}{l}23,5 \\
( \pm 6,0)\end{array}$ & $25,9( \pm 7,5)$ & $\begin{array}{l}\mathrm{PD} \geq 4 \mathrm{~mm}, \mathrm{CAL}(\geq 4 \mathrm{~mm}) \\
\text { y BOP. }\end{array}$ & $\begin{array}{l}\mathrm{PA} \geq 140 / 90 \mathrm{mmHg} \text { y } \\
\text { proteinuria } 2+\text { confirmada por } \\
\text { al menos } 0,3 \mathrm{~g} / 24 \mathrm{~h} \text {. }\end{array}$ \\
\hline 14 & $\begin{array}{l}\text { (Canakci } \\
\text { et al., 2004) }\end{array}$ & Turquía & 82 & $\begin{array}{l}25,9 \\
( \pm 5,9)\end{array}$ & $25,8( \pm 5,8)$ & $\begin{array}{l}\geq 4 \text { dientes con uno o más } \\
\text { sitios con } P D \geq 4 \mathrm{~mm} \text {, con } \\
\text { BOP y CAL } \geq 3 \mathrm{~mm} \text { en el } \\
\text { mismo sitio. }\end{array}$ & $\begin{array}{l}\mathrm{PA} \geq 140 / 90 \mathrm{mmHg} \text { en dos o } \\
\text { más ocasiones con un } \\
\text { intervalo de al menos } 4 \mathrm{~h} \mathrm{y} \\
\text { proteinuria } \geq 300 \mathrm{mg} / 24 \mathrm{~h} \text { o } \\
\text { cuando hubo un valor } 1+(30 \\
\text { mg/dL) en dos ocasiones } \\
\text { separadas con al menos } 6 \mathrm{~h} \text { de } \\
\text { diferencia, que se desarrolla } \\
\text { después de las } 20 \text { semanas de } \\
\text { gestación. }\end{array}$ \\
\hline 15 & $\begin{array}{l}\text { (Varshney } \\
\text { \& Gautam, } \\
\text { 2014) }\end{array}$ & India & 40 & 28,52 & $2.8) \S$ & $\begin{array}{l}\mathrm{PD} \geq 4 \mathrm{~mm} \text { y } \mathrm{CAL} \geq 3 \mathrm{~mm} \\
\text { en el mismo sitio en al } \\
\text { menos } 4 \text { dientes diferentes } \\
\text { no vecinos. }\end{array}$ & $\begin{array}{l}\mathrm{PA} \geq 140 / 90 \mathrm{~mm} \mathrm{Hg} \text { en dos } \\
\text { ocasiones distintas después de } \\
\text { la semana } 20 \text { de gestación y } \\
\geq 1+\text { proteinuria. }\end{array}$ \\
\hline
\end{tabular}

(OHI) Índice de higiene bucal simplificado; (GI) Índice Gingival; (PD) Profundidad de Sondaje; (CAL) Pérdida de inserción clínica; (BOP) Sangrado al sondaje; (PA) Presión Arterial. (*) No reporta DE. (§) Edad media del total de la muestra. Fuente: Autores.

La Tabla 2 muestra los factores de riesgo estudiados por los 15 estudios evaluados en la presente revisión sistemática. De los factores de riesgo observados se puede evidenciar que la edad, paridad, y fumar fueron los factores de riesgo más prevalentes en los artículos mencionados, siendo que la edad fue un factor de confusión importante para 11 (73,33\%) de los estudios, seguido de la paridad, que estuvo controlado en $10(66,67 \%)$ de los estudios, el tercer factor de riesgo más importante fue el fumar, que fue controlado en $7(46,67 \%)$ de los estudios analizados, por otra parte variables como la raza fue un factor de riesgo estudiado en apenas 1 (6,67\%), así como la media de ingresos que también fue estudiada en apenas 1 (6,67\%) de los estudios realizados. 
Tabla 2. Factores de riesgo analizados en los estudios evaluados.

\begin{tabular}{|c|c|c|c|c|c|c|c|c|c|c|c|c|c|c|c|c|c|}
\hline \multirow{2}{*}{$\begin{array}{l}\text { Variables de } \\
\text { confusión }\end{array}$} & \multicolumn{15}{|c|}{ ID del Estudio } & \multirow{2}{*}{$\begin{array}{l}\text { Total de variables } \\
\text { controladas }\end{array}$} & \multirow[t]{2}{*}{$\%$} \\
\hline & 1 & 2 & 3 & 4 & 5 & 6 & 7 & 8 & 9 & 10 & 11 & 12 & 13 & 14 & 15 & & \\
\hline Edad & + & - & + & + & - & + & l & + & + & + & + & + & - & + & + & 11 & 73,33 \\
\hline Raza & I & l & l & + & I & - & l & I & / & I & l & l & I & - & l & 1 & 6,67 \\
\hline Paridad & + & + & + & + & l & - & / & + & + & + & - & + & - & + & + & 10 & 66,67 \\
\hline Fumar & / & l & + & + & + & - & / & / & + & - & + & + & / & + & - & 7 & 46,67 \\
\hline Alcohol & I & l & l & + & - & - & l & I & - & - & l & + & I & - & I & 2 & 13,33 \\
\hline Ingresos & - & I & + & - & l & - & / & - & - & I & l & I & l & - & I & 1 & 6,67 \\
\hline Educación & - & / & + & / & / & / & / & - & + & - & + & + & - & - & I & 4 & 26,67 \\
\hline $\begin{array}{l}\text { Semanas de } \\
\text { gestación }\end{array}$ & - & I & l & - & - & + & l & I & I & I & - & l & I & + & I & 2 & 13,33 \\
\hline IMC & - & I & + & I & I & I & I & I & + & I & + & I & I & I & I & 3 & 20 \\
\hline Hipertensión & l & I & - & / & l & l & l & I & - & + & l & + & / & / & + & 3 & 20 \\
\hline
\end{tabular}

(+) Factor de confusión controlado; (-) Factor de confusión no controlado; (/): No reportado. (IMC) Índice de masa corporal. Fuente: Autores.

En la Tabla 3 se puede observar los resultados de los análisis de asociación existentes entre la enfermedad periodontal y preeclampsia, la misma que resulta positiva en 13 de los $15(86,67 \%)$ estudios. Mientras que en los 2 estudios restantes la asociación no se mostró estadísticamente significativa. 
Tabla 3. Odds Ratios y conclusiones de los estudios incluídos.

\begin{tabular}{|c|c|c|c|}
\hline ID & OR & IC $(95 \%)$ & Conclusiones principales de los autores \\
\hline 1 & 5,5 & $2,7-11,4$ & La enfermedad periodontal es más prevalente en mujeres con HP. \\
\hline 2 & 9,33 & $2,84-30,60$ & La periodontitis materna se asoció con la preeclampsia. \\
\hline 3 & 8,6 & $3,92-18,88$ & $\begin{array}{l}\text { Dentro de la población estudiada, la periodontitis fue un factor de riesgo para la } \\
\text { preeclampsia. }\end{array}$ \\
\hline 4 & 1,13 & $0,59-2,17$ & $\begin{array}{l}\text { Este estudio no apoya la hipótesis de una asociación entre enfermedad periodontal y } \\
\text { preeclampsia. }\end{array}$ \\
\hline 5 & 6,6 & $1,25-41,61$ & $\begin{array}{l}\text { Estos resultados indican que la preeclampsia podría estar asociada con la condición } \\
\text { periodontal materna y la limpieza interdental. }\end{array}$ \\
\hline 6 & 3,73 & $1,32-10,58$ & $\begin{array}{l}\text { La periodontitis se relacionó clínicamente con la preeclampsia; sin embargo, el proceso } \\
\text { inflamatorio sistémico que involucra a las citocinas TNF } \alpha \text { e IL-6 en presencia de } \\
\text { enfermedad periodontal, no pudo ser confirmado. }\end{array}$ \\
\hline 7 & $3,00 *$ & $1,91-4,87^{*}$ & $\begin{array}{l}\text { Los casos preeclámpticos tuvieron significativamente mayor pérdida de inserción y } \\
\text { recesión gingival que el grupo de control. }\end{array}$ \\
\hline 8 & 20,15 & $4,55-89,29$ & $\begin{array}{l}\text { La periodontitis tanto en el momento de la inscripción, como dentro de las } 48 \text { horas } \\
\text { posteriores al parto, puede estar asociada con un mayor riesgo de preeclampsia. }\end{array}$ \\
\hline 9 & 0,92 & $0,26-3,28$ & $\begin{array}{l}\text { Este estudio no proporciona evidencia convincente de que la enfermedad periodontal esté } \\
\text { asociada con el riesgo de preeclampsia entre las mujeres tailandesas. }\end{array}$ \\
\hline 10 & 1,52 & $1,01-2,29$ & $\begin{array}{l}\text { La periodontitis materna es un factor de riesgo asociado a la preeclampsia, enfatizando la } \\
\text { importancia de la atención periodontal en los programas prenatales. }\end{array}$ \\
\hline 11 & 7,9 & $1,9-32,8$ & $\begin{array}{l}\text { Las mujeres caucásicas con antecedentes recientes de preeclampsia de inicio temprano } \\
\text { tienen una peor condición periodontal, en comparación con las mujeres con partos sin } \\
\text { complicaciones. }\end{array}$ \\
\hline 12 & 1,88 & $1,15-3,06$ & $\begin{array}{l}\text { Se determinó que la periodontitis materna está asociada con un mayor riesgo de } \\
\text { preeclampsia. }\end{array}$ \\
\hline 13 & $4,1^{*}$ & $1,5-11,5^{*}$ & $\begin{array}{l}\text { La enfermedad periodontal crónica y la presencia de P. gingivalis, T. forsythia y E. } \\
\text { corrodens se asociaron significativamente con la preeclampsia en mujeres embarazadas. }\end{array}$ \\
\hline 14 & 3,47 & $1,07-11,95$ & $\begin{array}{l}\text { La enfermedad periodontal durante el embarazo se asocia con un mayor riesgo de } \\
\text { desarrollo de preeclampsia. }\end{array}$ \\
\hline 15 & 4,33 & $1,15-16,32$ & Se determinó que el estado oral materno se asocia con un mayor riesgo de preeclampsia. \\
\hline
\end{tabular}

(*) OR crudo. (OR) Odds ratio. (HP) Hipertensión del embarazo.

Fuente: Autores.

\section{Discusión}

De los datos extraídos de la Tabla 1, se evidencia que existe gran cantidad de estudios que analizan la posible asociación entre periodontitis y preeclampsia en países en vías de desarrollo, este fenómeno podría atribuirse al limitado acceso a los sistemas de salud en los países en donde fueron conducidos estos estudios, esta hipótesis podría ser respaldada por datos aportados por la Organización Mundial de la Salud (De Jesús-García et al., 2018) la incidencia de preeclampsia de casi 7 veces mayor en los países en desarrollo a comparación de los países desarrollados. Así mismo, Wei et al., (2013) en su estudio sugiere que la mayor incidencia de preeclampsia en países en desarrollo puede deberse al poco acceso a la atención dental, sin embargo, no pudo obtener datos relevantes que confirmen esta hipótesis.

Igualmente de los resultados obtenidos, podemos observar que si bien no existe una homogeneidad en la definición de periodontitis, la mayoría de los estudios que conforman nuestra revisión consideran un CAL $\geq 3 \mathrm{~mm}$ (Varshney \& Gautam, 2014)'(Desai et al., 2015)'(Moura da Silva et al., 2012)-(Canakci et al., 2004) y un PD $\geq 4$ mm (Varshney \& Gautam, 2014)'(Desai et al., 2015)'(Lohsoonthorn et al., 2009)'(Moura da Silva et al., 2012)'(Shetty et al., 2010)'(Contreras et al., 2006) como parámetros para definir esta enfermedad, aunque autores como Lohsoonthorn et al., (2009) y Contreras et al., (2006) consideran valores más elevados de CAL para la determinación de periodontitis. A su vez, estas mismas divergencias entre los 
parámetros que determinan la periodontitis también fueron observadas en estudios similares que abordaron este tema (Huang et al., 2014)'(Konopka \& Zakrzewska, 2020)'(Kunnen et al., 2010)'(Sgolastra et al., 2013). En el estudio conducido por Kunnen et al., (2010) debido a la elevada heterogeneidad de los criterios diagnósticos de la periodontitis, no se pudo aplicar métodos estadísticos, obteniendo al menos 9 definiciones distintas de periodontitis en 15 estudios revisados.

Por otro lado, en lo referente a los parámetros utilizados para determinar la preeclampsia, todos los autores aquí citados determinan una $\mathrm{PA} \geq 140 / 90 \mathrm{mmHg}$ como signo de preeclampsia, sin embargo, en lo referente a la proteinuria existen algunas divergencias, ya que algunos autores consideran un valor 2+ (Moura da Silva et al., 2012)(Kunnen et al., 2007)'(Contreras et al., 2006) y otros autores 1+ (Varshney \& Gautam, 2014)'(Lohsoonthorn et al., 2009)'(Taghzouti et al., 2012)'(Sayar et al., 2011)'(Cota et al., 2006)'(Canakci et al., 2004)'(Ha et al., 2011) en tiras reactivas respectivamente. Además, a pesar de que la mayoría de los estudios en nuestra investigación toman en cuenta la proteinuria como parte del diagnóstico de preeclampsia, en el año 2013 el Colegio Americano de Ginecología y Obstetricia (ACOG), indicó que una paciente con hipertensión después de las 20 semanas, aún sin proteinuria, debe ser considerado un caso de preeclampsia, pero en presencia de otros signos de severidad como trombocitopenia, alteración hepática, creatinina $>1,1 \mathrm{mg} / \mathrm{dL}$, edema pulmonar o sintomatología de preeclampsia (Pereira Calvo et al., 2020).

De los datos obtenidos, en la Tabla 2 se puede observar que uno de los factores de riesgo más relevantes fue la edad materna, ya que de los 15 estudios que conforman la revisión sistemática, 11 de ellos reportan datos de esta variable. Esto es apoyado por Das et al., (2019) el cual en su estudio retrospectivo que incluyó un total de 4820 partos, analizó la incidencia y factores de riesgo de la preeclampsia, demostrando que una edad materna mayor a 35 años representa un factor de riesgo para desarrollar preeclampsia.

Además, en nuestro estudio se informó que 10 artículos reportan la paridad como otro factor de riesgo importante para el desarrollo preeclampsia. Esto es apoyado igualmente por Das et al., (2019) que identificó que la probabilidad de desarrollar preeclampsia fue 2 veces mayor en mujeres primíparas en comparación con mujeres multíparas (OR 2,12, IC del 95\%: 1,25$3,60)$; por lo tanto, se puede suponer que la primiparidad puede ser un determinante de preeclampsia.

Otro factor de riesgo controlado en varios de los artículos presentados en la tabla 2 fue el hábito de fumar durante el embarazo. Dentro de los estudios incluidos, Siqueira et al., (2008) y Cota et al., (2006) reportaron que fumar durante el embarazo representó un menor riesgo para el desarrollo de preeclampsia, por lo que podría se podría tratar de un posible efecto protector. Esta hipótesis puede ser respaldada por la reciente revisión sistemática y metaanálisis publicada por Wang et al., (2021) que incluyó 13 estudios (9 cohorte, 4 casos-controles) con una población total de 3457 593, en la cual demostró que fumar durante el periodo de gravidez estuvo relacionado con un menor riesgo de preeclampsia (OR 0,65, IC del 95\%: 0,580,73. Se sugiere que la acción de la nicotina puede inhibir la interleucina-2 y la producción del factor de necrosis tumoral producido por células mononucleares (Dekker \& Sibai, 2001). Sin embargo, se debe recalcar los efectos nocivos de fumar durante el embarazo, como: retraso en el crecimiento uterino, riesgo de parto prematuro, alteraciones en el desarrollo del feto, etc, (Collet \& Beillard, 2005). Además, afecta la salud oral, produciendo desde cambios en el color de las restauraciones dentales, enfermedad periodontal, hasta lesiones potencialmente malignas y cáncer bucal (Komar et al., 2018).

En nuestra revisión 13 de los 15 estudios incluidos (86,67\%) mostraron una asociación positiva entre periodontitis y preeclampsia (Tabla 3), dichos datos coinciden con los resultados obtenidos por Konopka y Zakrzewska (2020) en su revisión sistemática publicada en el año 2020, en la cual se incluyeron 6 estudios de cohorte y 3 ensayos controlados aleatorizados (ECA), demostraron una asociación positiva entre la periodontitis y el riesgo de desarrollar preeclampsia en 5 de los 6 ensayos de cohorte, en los 3 ECA no se demostraron los beneficios del tratamiento periodontal (raspado y alisado radicular) en la prevención de la preeclampsia. 
Kunnen et al., (2010) en su revisión sistemática publicada en el año 2010, incluyó 12 estudios observacionales y 3 ECA. De los estudios observacionales, 8 mostraron una asociación positiva entre la enfermedad periodontal y preeclampsia, a diferencia de los otros 4 estudios, sin embargo, no pudieron aplicar métodos estadísticos debido a la heterogeneidad en las definiciones de enfermedad periodontal. Por otra parte, ninguno de los ECA reportó mejoras en la condición de salud de las mujeres preeclámpticas después del tratamiento periodontal durante el embarazo, por lo tanto, sugieren que la relación es cuestionable.

Por otro lado, Conde-Agudelo et al., (2008) en su revisión sistemática obtuvo 7 estudios de casos y controles y 2 estudios de cohortes, sin embargo, para su metaanálisis se incluyeron 6 estudios con criterios de diagnóstico bien definidos de enfermedad periodontal, esto permitió concluir que, las mujeres con enfermedad periodontal tenían un riesgo mayor de sufrir preeclampsia en comparación con las mujeres sin enfermedad periodontal (OR 1,76, IC del 95\%: 1,43-2,18).

Dentro de las fortalezas de nuestra investigación se destaca que los estudios fueron realizados en diferentes países, lo que podría producir que los resultados obtenidos sean más generalizables y al uso de la declaración STROBE (Vandenbroucke et al., 2009) para controlar la calidad de los artículos incluidos. Se decidió no incluir estudios realizados en pacientes con antecedentes de enfermedades sistémicas y limitar la edad de las mujeres embarazadas para poder controlar la confusión que podrían generar.

Se recomienda cautela a la hora de interpretar los resultados de esta investigación, debido a los diferentes criterios diagnósticos utilizados para periodontitis, al carácter multifactorial de periodontitis y preeclampsia, el momento exacto del examen clínico, la variabilidad de los factores de confusión controlados entre cada estudio y a variables que no fueron controladas. Además, se resalta que en los estudios de Sayar et al., (2011) y Contreras et al., (2006) los cuales reportaron una asociación positiva entre enfermedad periodontal y preeclampsia (OR 4,1, IC del 95\%: 1,5-11,5 y OR 3,0, IC del 95\%: 1,914,87 respectivamente) se determinaron datos sociodemográficos, sin embargo, los autores no informaron haber realizado ajustes por factores de confusión.

\section{Conclusión}

En base a los resultados obtenidos y a que la preeclampsia y eclampsia son una de las cuatro causas de mortalidad en el periodo de gravidez, parto o post parto (Condo-Baque et al., 2018). En nuestro estudio se enfatiza la importancia de las implicaciones de la periodontitis en el embarazo, y se promueve la implementación de atención periodontal en los programas prenatales.

Para futuros trabajos de investigación de la asociación entre estas patologías se recomienda utilizar los criterios diagnósticos actuales de periodontitis (Herrera et al., 2018) para de esta forma puedan obtenerse estudios más homogéneos que puedan ser analizados estadísticamente y proporcionen datos más confiables. Se incita a realizar más investigaciones que puedan ayudar a comprender mejor el efecto del tratamiento periodontal en las mujeres embarazadas y que aclaren el posible mecanismo de asociación entre estas dos patologías.

\section{Referencias}

Barak, S., Oettinger-Barak, O., Machtei, E. E., Sprecher, H., \& Ohel, G. (2007). Evidence of Periopathogenic Microorganisms in Placentas of Women With Preeclampsia. Journal of Periodontology, 78(4), 670-676. https://doi.org/10.1902/jop.2007.060362

Boggess, K. A., Lieff, S., Murtha, A. P., Moss, K., Beck, J., \& Offenbacher, S. (2003). Maternal periodontal disease is associated with an increased risk for preeclampsia. Obstetrics and Gynecology, 101(2), 227-231. https://doi.org/10.1016/S0029-7844(02)02314-1

Canakci, V., Canakci, C. F., Canakci, H., Canakci, E., Cicek, Y., Ingec, M., Ozgoz, M., Demir, T., Dilsiz, A., \& Yagitz, H. (2004). Periodontal Disease as a Risk Factor for Preeclampsia. Australian and New Zealand Journal of Obstetrics and Gynaecology, 1(1), 568-573. https://doi.org/10.17795/intjsh-18908

Carvajal, P. (2016). Enfermedades periodontales como un problema de salud pública: el desafío del nivel primario de atención en salud. Revista Clínica de 
Periodoncia, Implantología y Rehabilitación Oral, 9(2), 177-183. https://doi.org/10.1016/j.piro.2016.07.001

Casas, A. (2020). Nueva clasificación de enfermedades periodontales y periimplantarias. Revista el dentista moderno, 28-41. https://www.eldentistamoderno.com/wp-content/uploads/pdf/DM48-pag28-41.pdf

Collet, M., \& Beillard, C. (2005). Conséquences du tabagisme sur le développement fotal et le risque de retard de croissance intra-utérin ou de mort fœtale in utero. J Gynecol Obstet Biol Reprod, 34(1), 135-145. https://doi.org/10.4324/9781843141051-5

Conde-Agudelo, A., Villar, J., \& Lindheimer, M. (2008). Maternal infection and risk of preeclampsia: Systematic review and metaanalysis. American Journal of Obstetrics and Gynecology, 198(1), 7-22. https://doi.org/10.1016/j.ajog.2007.07.040

Condo-Baque, C., Barreto-Pincay, G., Montaño-Parrales, G., Borbor-Sánchez, L., Manrique-Regalado, G., \& García-Sigcha, A. (2018). Preeclampsia y eclampsia en pacientes atendidas en el área de emergencia del Hospital Verdi Cevallos Balda julio 2016 - junio del 2017. Revista Cientifica Dominio de Las Ciencias, 4(3), 278-293. https://doi.org/http://dx.doi.org/10.23857/dc.v4i3.810

Contreras, A., Herrera, J. A., Soto, J. E., Arce, R. M., Jaramillo, A., \& Botero, J. E. (2006). Periodontitis Is Associated With Preeclampsia in Pregnant Women. Journal of Periodontology, 77(2), 182-188. https://doi.org/10.1902/jop.2006.050020

Cota, L. O. M., Guimarães, A. N., Costa, J. E., Lorentz, T. C., \& Costa, F. O. (2006). Association Between Maternal Periodontitis and an Increased Risk of Preeclampsia. Journal of Periodontology, 77(12), 2063-2069. https://doi.org/10.1902/jop.2006.060061

Das, S., Das, R., Bajracharya, R., Baral, G., Jabegu, B., Odland, J. Ø., \& Odland, M. L. (2019). Incidence and risk factors of pre-eclampsia in the paropakar maternity and women's hospital, Nepal: A retrospective study. International Journal of Environmental Research and Public Health, 16(19), 1-8. https://doi.org/10.3390/ijerph16193571

De Jesús-García, A., Jimenez-Baez, M. V., González-Ortiz, D. G., De la Cruz-Toledo, P., Kuc-Peña, L. M., \& Sandoval-Jurado, L. (2018). Características clínicas, epidemiológicas y riesgo obstétrico de pacientes con preeclampsia-eclampsia. Rev. enferm. Inst. Mex. Seguro Soc, $26(4)$, $256-262$. https://www.medigraphic.com/pdfs/enfermeriaimss/eim-2018/eim184e.pdf

Dekker, G., \& Sibai, B. (2001). Primary, secondary, and tertiary prevention of pre-eclampsia. The Lancet, 357, 209-215. https://doi.org/10.1016/S01406736(00)03599-6

Desai, K., Desai, P., Duseja, S., Kumar, S., Mahendra, J., \& Duseja, S. (2015). Significance of maternal periodontal health in preeclampsia. Journal of International Society of Preventive and Community Dentistry, 5(2), 103-107. https://doi.org/10.4103/2231-0762.155734

Ha, J. E., Oh, K. joon, Yang, H. J., Jun, J. K., Jin, B. H., Paik, D. Il, \& Bae, K. H. (2011). Oral Health Behaviors, Periodontal Disease, and Pathogens in Preeclampsia: A Case-Control Study in Korea. Journal of Periodontology, 82(12), 1685-1692. https://doi.org/10.1902/jop.2011.110035

Herrera, D., Figuero, E., Shapira, L., Jin, L., \& Sanz, M. (2018). La nueva clasificación de las enfermedades periodontales. Revista Científica de la Sociedad Española de Periodoncia, 11. http://www.sepa.es/web_update/wp-content/uploads/2018/10/p11ok.pdf

Huang, X., Wang, J., Liu, J., Hua, L., Zhang, D., Hu, T., \& Ge, Z. li. (2014). Maternal periodontal disease and risk of preeclampsia: A meta-analysis. Journal of Huazhong University of Science and Technology - Medical Science, 34(5), 729-735. https://doi.org/10.1007/s11596-014-1343-8

Komar, K., Glavina, A., Boras, V. V., Verzak, Ž., \& Brailo, V. (2018). Impact of smoking on oral health: Knowledge and attitudes of dentists and dental students. Acta Stomatologica Croatica, 52(2), 148-155. https://doi.org/10.15644/asc52/2/8

Konopka, T., \& Zakrzewska, A. (2020). Periodontitis and risk for preeclampsia - A systematic review. Ginekologia Polska, 91(3), 158-164. https://doi.org/10.5603/GP.2020.0024

Kunnen, A., Blaauw, J., Van Doormaal, J. J., Van Pampus, M. G., Van Der Schans, C. P., Aarnoudse, J. G., Van Winkelhoff, A. J., \& Abbas, F. (2007). Women with a recent history of early-onset pre-eclampsia have a worse periodontal condition. Journal of Clinical Periodontology, 34(3), 202-207. https://doi.org/10.1111/j.1600-051X.2006.01036.x

Kunnen, A., Van Doormaal, J. J., Abbas, F., Aarnoudse, J. G., Van Pampus, M. G., \& Faas, M. M. (2010). Periodontal disease and pre-eclampsia: A systematic review. Journal of Clinical Periodontology, 37(12), 1075-1087. https://doi.org/10.1111/j.1600-051X.2010.01636.x

Lohsoonthorn, V., Kungsadalpipob, K., Chanchareonsook, P., Limpongsanurak, S., Vanichjakvong, O., Sutdhibhisal, S., Sookprome, C., Wongkittikraiwan, N., Kamolpornwijit, W., Jantarasaengaram, S., Manotaya, S., Siwawej, V., Barlow, W. E., Fitzpatrick, A. L., \& Williams, M. A. (2009). Maternal periodontal disease and risk of preeclampsia: A case-control study. American Journal of Hypertension, 22(4), 457-463. https://doi.org/10.1038/ajh.2008.365

Macedo, T. C. C., Montagna, E., Trevisan, C. M., Zaia, V., De Oliveira, R., Barbosa, C. P., Laganà, A. S., \& Bianco, B. (2020). Prevalence of preeclampsia and eclampsia in adolescent pregnancy: A systematic review and meta-analysis of 291,247 adolescents worldwide since 1969. European Journal of Obstetrics and Gynecology and Reproductive Biology, 248, 177-186. https://doi.org/10.1016/j.ejogrb.2020.03.043

Moura da Silva, G., Coutinho, S. B., Piscoya, M. D. B., Ximenes, R. A., \& Jamelli, S. R. (2012). Periodontitis as a Risk Factor for Preeclampsia. Journal of Periodontology, 83(11), 1388-1396. https://doi.org/10.1902/jop.2012.110256

Nazir, M., Al-Ansari, A., Al-Khalifa, K., Alhareky, M., Gaffar, B., \& Almas, K. (2020). Global Prevalence of Periodontal Disease and Lack of Its Surveillance. The Scientific World Journal. https://doi.org/10.1155/2020/2146160

Nocini, R., Lippi, G., \& Mattiuzzi, C. (2020). Periodontal disease: the portrait of an epidemic. Journal of Public Health and Emergency, 4(10). https://doi.org/10.21037/jphe.2020.03.01

OMS. (2014). Recomedaciones de la OMS para la prevención y el tratamiento de la preeclmpsia y la eclampsia. 1-38. http://apps.who.int/iris/bitstream/10665/138405/1/9789243548333_spa.pdf?ua=1\&ua=1 
Pereira Calvo, J., Pereira Rodríguez, Y., \& Quirós Figueroa, L. (2020). Actualización en preeclampsia (Update in preeclampsia). Revista Médica Sinergia, 5(1). https://doi.org/https://doi.org/10.31434/rms.v5i1.340

Politano, G. T., Passini, R., Nomura, M. L., Velloso, L., Morari, J., \& Couto, E. (2011). Correlation between periodontal disease, inflammatory alterations and pre-eclampsia. Journal of Periodontal Research, 46(4), 505-511. https://doi.org/10.1111/j.1600-0765.2011.01368.x

Pralhad, S., Thomas, B., \& Kushtagi, P. (2013). Periodontal Disease and Pregnancy Hypertension: A Clinical Correlation. Journal of Periodontology, 84(8), 1118-1125. https://doi.org/10.1902/jop.2012.120264

Sayar, F., Sadat Hoseini, M., \& Abbaspour, S. (2011). Effect of periodontal disease on preeclampsia. Iranian Journal of Public Health, 40(3), 122-127. https://www.ncbi.nlm.nih.gov/pmc/articles/PMC3481644/

Sgolastra, F., Petrucci, A., Severino, M., Gatto, R., \& Monaco, A. (2013). Relationship between periodontitis and pre-eclampsia: a meta-analysis. PLOS ONE, 8(8), 24-26. https://doi.org/10.1371/journal.pone.0071387

Shetty, M., Shetty, P. K., Ramesh, A., Thomas, B., Prabhu, S., \& Rao, A. (2010). Periodontal disease in pregnancy is a risk factor for preeclampsia. Acta Obstetricia et Gynecologica Scandinavica, 89(5), 718-721. https://doi.org/10.3109/00016341003623738

Siqueira, F. M., Miranda Cota, L. O., Costa, J. E., Amaral Haddad, J. P., Quintão Lana, Â. M., \& Oliveira Costa, F. (2008). Maternal Periodontitis as a Potential Risk Variable for Preeclampsia: A Case-Control Study. Journal of Periodontology, 79(2), 207-215. https://doi.org/10.1902/jop.2008.070174

Taghzouti, N., Xiong, X., Gornitsky, M., Chandad, F., Voyer, R., Gagnon, G., Leduc, L., Xu, H., Tulandi, T., Wei, B., Sénécal, J., Velly, A. M., Salah, M. H., \& Fraser, W. D. (2012). Periodontal Disease is Not Associated With Preeclampsia in Canadian Pregnant Women. Journal of Periodontology, 83(7), 871-877. https://doi.org/10.1902/jop.2011.110342

Urrutia, G., \& Bonfill, X. (2010). Declaración PRISMA: una propuesta para mejorar la publicación de revisiones sistemáticas y metaanálisis. Medicina Clínica, 135(11), 507-511. https://doi.org/https://doi.org/10.1016/j.medcli.2010.01.015

Vandenbroucke, J. P., Von Elm, E., Altman, D. G., Gøtzsche, P. C., Mulrow, C. D., Pocock, S. J., Poole, C., Schlesselman, J. J., \& Egger, M. (2009). Mejorar la comunicación de estudios observacionales en epidemiología (STROBE): explicación y elaboración. Gaceta Sanitaria, 23(2), 1-28. https://doi.org/10.1016/j.gaceta.2008.12.001

Varshney, S., \& Gautam, A. (2014). Poor periodontal health as a risk factor for development of pre-eclampsia in pregnant women. Journal of Indian Society of Periodontology, 18(3), 321-325. https://doi.org/10.4103/0972-124X.134569

Wang, J., Yang, W., Xiao, W., \& Cao, S. (2021). The association between smoking during pregnancy and hypertensive disorders of pregnancy: A systematic review and meta-analysis. International Journal of Gynecology \& Obstetrics, 00, 1-11. https://doi.org/10.1002/ijgo.13709

Wei, B. J., Chen, Y. J., Yu, L., \& Wu, B. (2013). Periodontal Disease and Risk of Preeclampsia: A Meta-Analysis of Observational Studies. PLOS ONE, 8(8), 1-6. https://doi.org/10.1371/journal.pone.0070901 